Inference of ICF Implosion Core Mix using Experimental Data and Theoretical Mix Modeling

L. Welser-Sherrill, D. A. Haynes, R. C. Mancini, J. H. Cooley, R. Tommasini, I. E. Golovkin, M. E. Sherrill, S. W. Haan

May 28, 2008

High Energy Density Physics 
This document was prepared as an account of work sponsored by an agency of the United States government. Neither the United States government nor Lawrence Livermore National Security, LLC, nor any of their employees makes any warranty, expressed or implied, or assumes any legal liability or responsibility for the accuracy, completeness, or usefulness of any information, apparatus, product, or process disclosed, or represents that its use would not infringe privately owned rights. Reference herein to any specific commercial product, process, or service by trade name, trademark, manufacturer, or otherwise does not necessarily constitute or imply its endorsement, recommendation, or favoring by the United States government or Lawrence Livermore National Security, LLC. The views and opinions of authors expressed herein do not necessarily state or reflect those of the United States government or Lawrence Livermore National Security, LLC, and shall not be used for advertising or product endorsement purposes. 


\title{
Inference of ICF Implosion Core Mix using Experimental Data and Theoretical Mix Modeling
}

\author{
L. Welser-Sherrill, ${ }^{1}$ D. A. Haynes,${ }^{1}$ R. C. Mancini,${ }^{2}$ J. H. Cooley, ${ }^{1}$ \\ R. Tommasini, ${ }^{3}$ I. E. Golovkin, ${ }^{4}$ M. E. Sherrill, ${ }^{1}$ and S. W. $\operatorname{Haan}^{3}$ \\ ${ }^{1}$ Los Alamos National Laboratory, New Mexico, 87545 \\ ${ }^{2}$ Department of Physics, University of Nevada, Reno, Nevada, 89557 \\ ${ }^{3}$ Lawrence Livermore National Laboratory, Livermore, California, 94550 \\ ${ }^{4}$ Prism Computational Sciences, Madison, Wisconsin, 53703
}

(Dated: May 19, 2008)

\begin{abstract}
The mixing between fuel and shell materials in Inertial Confinement Fusion (ICF) implosion cores is a current topic of interest. The goal of this work was to design direct-drive ICF experiments which have varying levels of mix, and subsequently to extract information on mixing directly from the experimental data using spectroscopic techniques. The experimental design was accomplished using hydrodynamic simulations in conjunction with Haan's saturation model, which was used to predict the mix levels of candidate experimental configurations. These theoretical predictions were then compared to the mixing information which was extracted from the experimental data, and it was found that Haan's mix model performed well in predicting trends in the width of the mix layer. With these results, we have contributed to an assessment of the range of validity and predictive capability of the Haan saturation model, as well as increased our confidence in the methods used to extract mixing information from experimental data.

PACS numbers: 52.57.-z, 52.70.-m, 52.57.Fg
\end{abstract}

\section{INTRODUCTION}

The mixing between fuel and shell materials in ICF implosion cores is a topic of current interest. Mixing due to hydrodynamic instabilities can affect implosion dynamics and could also go so far as to prevent ignition [1]. The ultimate goal of this work is to facilitate a more complete understanding of the mixing process by validating both experimentally- and theoretically-based mix diagnostic tools.

In previous work, we have demonstrated that it is possible to extract information on mixing directly from experimental data using spectroscopic techniques [2-4]. The development was first carried out based on a set of indirect drive implosions at the Laboratory for Laser Energetics' OMEGA laser [5]. In order to compare the datadriven analysis to a theoretical framework, two independent mix models, Youngs' phenomenological model [6] and the Haan saturation model [7], were previously implemented in conjunction with a series of clean hydrodynamic simulations which modeled the experiments in question [4]. It was found that Youngs' and Haan's mix models, as applied to the indirect drive case, provided results that demonstrated less mix than that derived from the experiment.

This paper will describe a new approach to address the problem just presented. An important outcome of the current work is an assessment of our ability to predict the extent of mix in ICF experiments by using a combination of hydrodynamic simulation post-processing and off-line mix modeling.

Theoretical mix modeling was used a priori to design direct drive experiments with measurably different levels of mix [8]. First a number of hydro simulations were post-processed with Haan's saturation model to study the parameter space, in order to determine which physical characteristics of the lasers and targets are most sensitive to mix. Next, detailed hydro simulations of the most appealing cases were performed to settle on a series of experimental conditions. The chosen designs consisted of three experimental configurations that were nominally identical except for the varying shell thickness of the plastic capsules.

The experiments were conducted based on these designs, and information on the mixing region was directly extracted from the experimental data using the narrowband image intensity analysis described in Ref. [2]. The data-derived spatial mixing profiles were evaluated to determine the corresponding width of the mixing region, for direct comparison to the mix modeling results.

\section{EXPERIMENTAL DESIGN PHASE}

The main goal in the initial phase was to design a sequence of direct drive ICF implosions which would exhibit measurably different levels of mixing, while simultaneously maintaining similar temperature and density conditions at peak x-ray emission [9]. This latter consideration was vital, since the experimental data analysis relies on narrow-band images integrated over 50 ps beginning roughly at the time of peak x-ray emission [10], and uses Ar K-shell x-ray line emission spectroscopy [11-21] to experimentally extract mixing data.

We began designing around the parameters of a socalled nominal direct drive implosion, the characteristics of which are shown in Fig. 1. This nominal implosion was originally designed to achieve electron temperatures and 
(a)

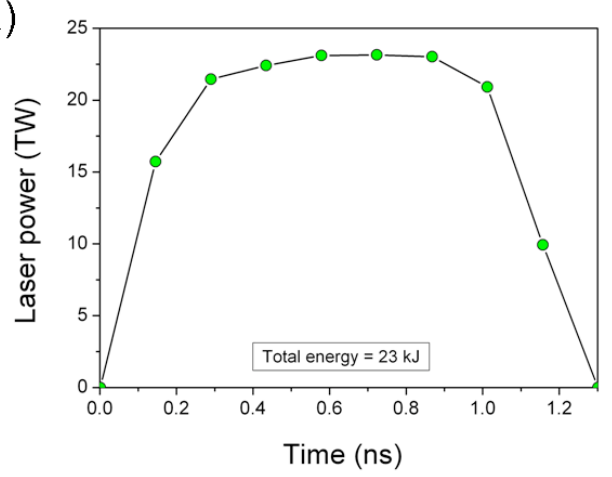

(b)

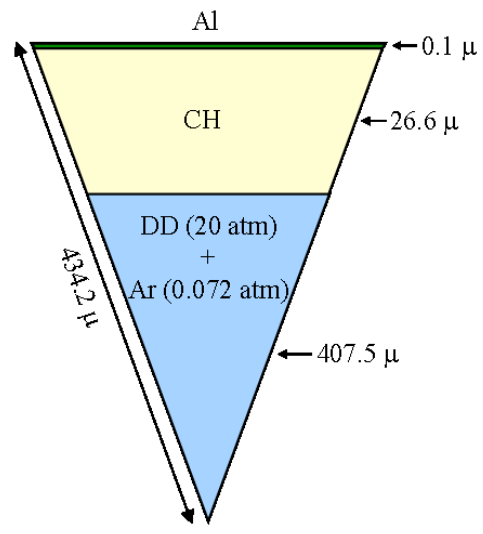

FIG. 1: (Color online) Characteristics of the nominal direct drive experiment used for design. (a) Nominal 1 ns square laser pulse taken from representative experiment. (b) Target cross-section, with materials and fill pressures noted.

densities that would be consistent with the conditions necessary to employ Ar K-shell x-ray spectroscopy [9]. By keeping the temperatures and densities approximately constant between shots, the idea was to isolate the experimental signal differences caused purely by mix amounts. In the design phase, mix was measured in terms of a mix width, which is defined as the physical extent of the layer that contains material from both the shell and the fuel [4].

\section{A. Radiation hydrodynamic simulations}

A number of experimental parameters were identified which could theoretically affect mix, including the laser pulse shape, duration, and energy; the gas and dopant fill pressures; the inner capsule radius; and the shell thickness. In order to maintain roughly the same temperature and density conditions as in the nominal case, only the laser energy, the fill gas amount, and the shell thickness were used as adjustable parameters in the design. Pulse shape and duration were not altered in the simulations due to the difficulty in changing these parameters from shot to shot. The fill gas material $\left(\mathrm{D}_{2}\right)$, and the dopant material (Ar) and corresponding concentration, were not altered. The shell material remained plastic for all simulations [9], and the outer Al layer, which helps minimize leakage of the gas, was not changed.

The experimental design work involved several steps. First, a number of 1d HELIOS [22, 23] radiation hydrodynamic simulations were performed to study the parameter space based on modifications to the nominal case shown in Fig. 1. The output from HELIOS, which is clean (includes no mix effects), was then post-processed with Haan's mix model to test the sensitivity to mix of the adjustable parameters being studied. Next, the parameter space was narrowed down to perform more detailed simulations of the parameters which appeared to be most sensitive to mix. Finally, in-depth modeling of the best pool of candidates for the design was performed with HELIOS-CR $[22,23]$ and was used to make a final determination for the experimental specifications.

\section{B. Mix modeling}

Each hydro simulation was post-processed using two independent mix models, Youngs' and Haan's, in order to extract a time-dependent mix width profile. Since a number of simulations had to be post-processed, IDL tools with graphical user interfaces were built for both Youngs' and Haan's models to systematically calculate the time-dependent mix widths [24]. The mix layer itself is defined as the sum of the widths of the bubble and spike regions, $h=h_{b}+h_{s}$, where the bubble represents the penetration of light fluid (fuel) into heavy, and the spike represents the penetration of heavy fluid (shell) into light.

Youngs' model [6], which is based on a phenomenological description of the growth of the mixing region as an effect of the Rayleigh-Taylor instability, was implemented using the same methodology as discussed in Ref. [4]. However, the mix widths calculated by this model did not demonstrate a significant sensitivity to variation in the experimental parameters; therefore, Youngs' model was not used to design the experiments.

The Haan saturation model [7] is based on the idea that instabilities are seeded by a number of experimental effects. If there are very minor initial seeds, then Youngs' phenomenological model accounts for the most important contributions. On the other hand, in an experimental situation such as laser-induced fusion, the initial perturbations can be significant and can arise from several factors. Haan's model calculates a mix width by estimating the growth of multi-mode initial perturbations on the fuelshell interface. For the direct drive case discussed here, the initial target surface roughness, the laser beam power imbalance, and the laser imprint spectra as a function of mode number were used. The modes grow exponentially until saturation occurs, at which time the mode growth becomes linear in time.

Each initial perturbation spectrum $\left|R_{l m}(0)\right|$ is sub- 
jected to the growth formula

$$
\left|R_{l m}\right|=e^{\xi t}\left|R_{l m}(0)\right|
$$

where

$$
\xi=\sqrt{g k A}
$$

is the growth factor, which is a function of the acceleration $g$, the wavenumber $k=\frac{2 \pi}{\lambda}$, and the Atwood number $A$. These terms are based on output from the hydro simulations, and are calculated as an average over the period of time from the beginning of the deceleration phase to the time step being evaluated. After the modal growth is accomplished, the perturbations are added in quadrature, producing a single $R_{l m}$ which is subjected to saturation modeling. If individual modes have saturated, the amplitudes are relaxed using the formula

$$
R_{l m}(l)=S_{l}\left[1+\ln \frac{R_{l m}}{S_{l}}\right],
$$

where

$$
S_{l}=\frac{2 R_{0}}{l^{2}}
$$

is the saturation level as a function of mode number $l$ [25], and $R_{0}$ is the capsule radius at the time step being calculated. The root mean square perturbation $\sigma$ is extracted from

$$
\sigma=\sqrt{\frac{1}{4 \pi} \sum_{i}(2 l+1) R_{l m}^{2}} .
$$

The total mix width $h$ is then calculated to be the sum of $h_{b}+h_{s}$,

$$
\begin{gathered}
h_{b}=\sqrt{2} \sigma \\
h_{s}=\sqrt{2} \sigma(1+A) .
\end{gathered}
$$

These mix width contributions are calculated for each time step in the simulation.

The prescription just given is called the nominal Haan model [7]. If a multiplicative factor (referred to here as the Haan multiplier) is applied to the initial perturbation spectra, the mix width can change dramatically. The nominal Haan model result is calculated with a multiplier of 1.0 .

A good way to use Haan's model is to think of the nominal result as a baseline, and to recognize that the mix width could increase (more likely) or decrease (less likely) based on additional experimental effects. It would not be unreasonable to consider that one or more of the initial perturbations are in fact enhanced by experimental imperfections. For example, in the previous work based on indirect drive experiments, the nominal Haan model result at the time the data were recorded was $4.1 \mathrm{mi}$ crons, while the experimentally-derived mix width was
$10 \pm 2$ microns. A multiplier of 2.7 would bring the Haan model results up to the level of the experimental data analysis [4]. That value for the multiplier would fold in any additional uncertainties in the initial perturbation amplitudes used by Haan's model. An interesting secondary goal of this new series of experiments is to investigate whether the same multiplicative scaling factor would apply to all direct drive designs, and if so, how that factor relates to the indirect drive multiplier. We will investigate this question in Section $\mathrm{V}$.

In general, the mix width predicted by Haan's model shows significant sensitivity to variation in the experimental design. This may be due to the fact that the model is based on experimentally derived perturbations and thus includes more effects than Youngs' phenomenological model.

\section{Parameter space study}

The initial parameter study was performed with HELIOS in order to determine which physical characteristics are most sensitive to mix. All simulations were ultimately compared to the nominal case described in Fig. 1. Simulations were grouped into clusters that shared common inquiries into the parameters. The following quantities were extracted from each simulation and used as input for the mix modeling, which provided mix width predictions for each candidate experimental design: core size, bang time (time of peak neutron production), peak compression (time at which the core is fully compressed), $T_{e}(r)$ (electron temperature profile at bang time and at peak compression of the capsule), $N_{e}(r)$ (electron density profile at bang time and at peak compression of the capsule), $v(t)$ (velocity of the fuel-shell interface as a function of time), $r(t)$ (radius as a function of time for all zones in the problem), $F(t)$ (net flux on the fuel-shell interface, used to calculate the time of peak Ar x-ray emission), $\rho_{\text {gas }}(t)$ (mass density of the last zone in the gas), $\rho_{\text {shell }}(t)$ (mass density of the first zone in the shell), and $A(t)$ (Atwood number based on the two zones on either side of the fuelshell interface).

The parameter study investigated the following changes from the nominal case: conserve mass in both gas and shell with the capsule radius, shell thickness, and fill pressure as variables; do not conserve mass in the gas and shell, instead correlate the radius and shell thickness; maintain the nominal inner radius and vary only the shell thickness; change the fill pressure only; change the total laser energy only.

The main goal was to search for experimental designs that yielded mix widths that were considerably different from that of the nominal case, but that retained approximately the same temperatures and densities [9]. This constrained the design significantly by only allowing small deviations in the experimental parameters.

For the early groups of simulations, it became apparent that changing the shell thickness (and keeping all other 
(a)

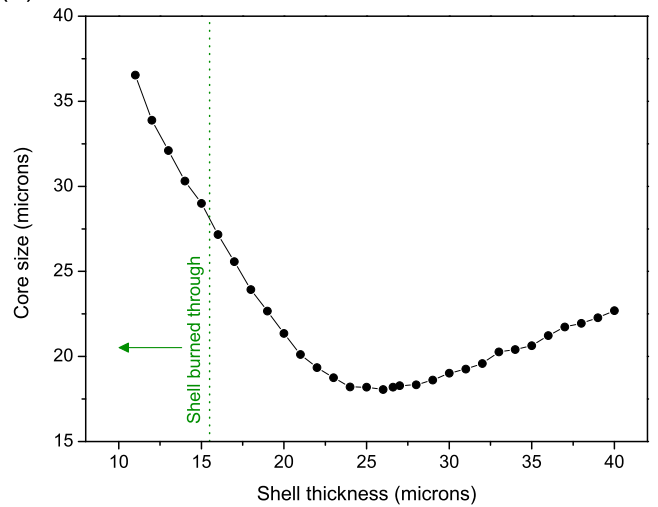

(b)

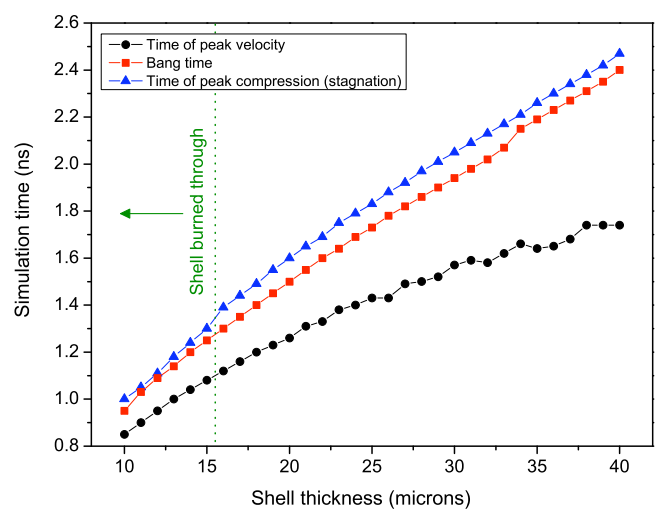

(c)

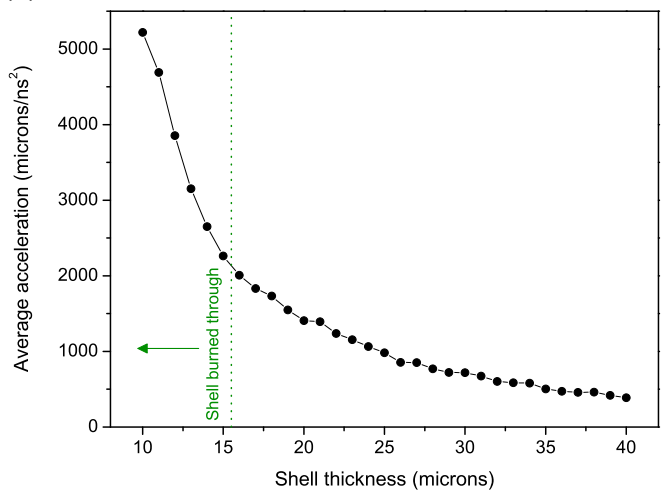

FIG. 2: (Color online) Data output from hydro simulations as a function of shell thickness. (a) Core size at peak compression, (b) timing, and (c) average acceleration through the deceleration phase. Shell thicknesses of less than $16 \mu \mathrm{m}$ showed signs of being burned through.

aspects of the nominal capsule identical) led to the most noticeable sensitivity to mix. Therefore, studies of the mix as a function of shell thickness were performed. In totality, the mix width was studied for shell thicknesses between 10 and 40 microns, with a step size of 1 micron between simulations.

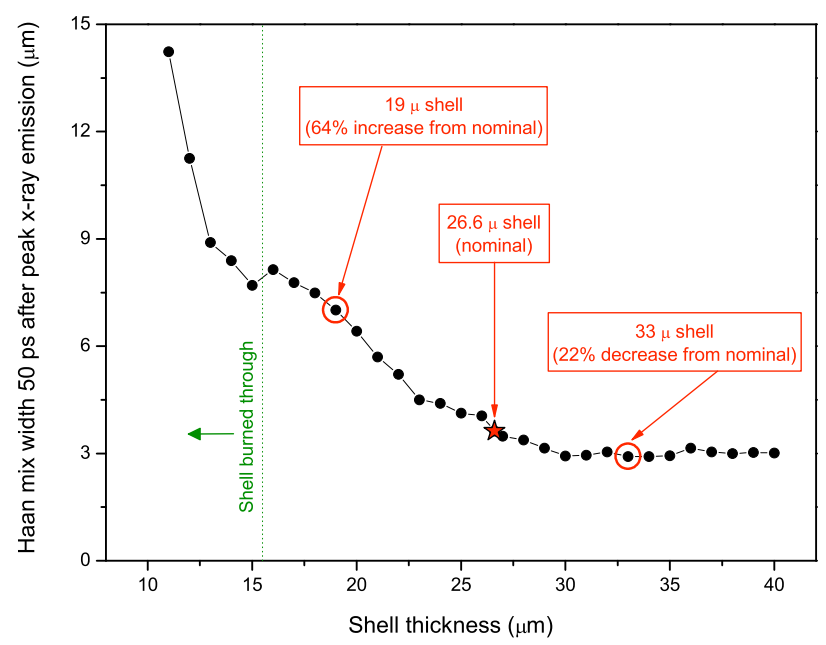

FIG. 3: (Color online) Mix widths at 50 ps after peak x-ray emission as a function of shell thickness, according to Haan's model. The chosen designs, 19, 26.6, and $33 \mu \mathrm{m}$ shells, are indicated.

Figure 2 shows a compilation of data output from the hydro simulations concentrating on changing shell thickness only. In Fig. 2(a), the core size decreases as the shell thickness increases, and it subsequently increases again around the nominal shell thickness of $26.6 \mu \mathrm{m}$. Figure 2(b) indicates that when the bang time and the peak compression time are nearly simultaneous, the shell experiences burn-through. When this occurs, there is no material left to continue the momentum inward, and therefore the core cannot continue to compress after peak burn. This was the case for shells with a thickness of 15 microns or less. Figure 2(c) plots the average acceleration, which is taken through the deceleration phase only up to $50 \mathrm{ps}$ after the time of peak x-ray emission [10], and is an important quantity used in Haan's model to calculate the growth rates.

We now focus in detail on the mixing post-processor results from the simulations based on changing shell thickness. Figure 3 shows the results of post-processing the hydro simulations with the Haan saturation model. It demonstrates that according to Haan's model, the shell thickness provides a significant lever arm for varying the amount of mix.

\section{Proposed experimental designs}

The sensitivity to mix provided by Haan's model and demonstrated in Fig. 3 facilitated the selection of several experimental designs. Two cases of interest are circled in Fig. 3: the 19 micron shell, which according to Haan's model exhibits $64 \%$ more mix than nominal, and the 33 micron shell, which displays $22 \%$ less mix than nominal. 
The volume- and time-averaged temperatures and densities were studied for these two cases, and were found to lie within the sensitivity range for Ar spectroscopy [9].

As a check on these preliminary findings, HELIOS-CR calculations using inline collisional-radiative atomic kinetic modeling were performed. HELIOS-CR, which uses NLTE physics, is generally expected to be more accurate than the baseline HELIOS LTE approach, because the atomic physics and radiation cooling effects of the Ar dopant can be more appropriately dealt with [22, 23]. These simulations took approximately 30 hours to run. It should be emphasized that we use HELIOS-CR only for an additional check: the fill conditions of the experiments were carefully conceived so that the Ar would have as little effect as possible on the implosion dynamics. Therefore, it was not surprising that the HELIOS-CR results were similar to the HELIOS results just discussed.

Table I presents the differing attributes of the three HELIOS-CR simulations. The laser (1 ns square, $23 \mathrm{~kJ}$ ), $\mathrm{D}_{2}$ fill pressure (20 atm), Ar fill pressure (0.18\%), and inner capsule radius $(407.5 \mu \mathrm{m})$ were identical for all simulations.

We settled on performing at least one experiment based on each of the designs described in Table I. All experiments were planned to have the same characteristics as the nominal experiment described in Fig. 1, with the exception of the thickness of the plastic shell.

\section{E. Sensitivity and tolerance studies}

Following the identification of the three experimental configurations discussed above, an investigation of the sensitivities and tolerances in the design was completed.

There are three perturbation spectra currently used in Haan's model for this direct drive case: target surface roughness, laser beam power imbalance, and laser imprint. The surface roughness spectrum has the smallest overall amplitude. Therefore, it should have the smallest effect on the overall calculation of the mix width. The power imbalance has the largest amplitude, especially at smaller mode numbers, and therefore should have the largest effect on mix.

According to Haan's prescription, the modes grow with an exponential growth factor. The saturation modeling is then performed for saturated modes. The composite spectrum, comprised of all three perturbation spectra

TABLE I: Experimental design characteristics and resulting mix predicted by Haan's model. Core size (interface position plus bubble width) and Haan mix values (bubble plus spike) are taken 50 ps after the time of peak x-ray emission [10].

\begin{tabular}{|c|c|c|c|}
\hline Shot type & Shell thickness & Core size & Haan mix \\
\hline Thin & $19.0 \mu \mathrm{m}$ & $35.1 \mu \mathrm{m}$ & $20.7 \mu \mathrm{m}$ (59\% of core) \\
Nominal & $26.6 \mu \mathrm{m}$ & $26.3 \mu \mathrm{m}$ & $4.2 \mu \mathrm{m}$ (16\% of core) \\
Thick & $33.0 \mu \mathrm{m}$ & $28.3 \mu \mathrm{m}$ & $2.8 \mu \mathrm{m}$ (10\% of core) \\
\hline
\end{tabular}

(a)

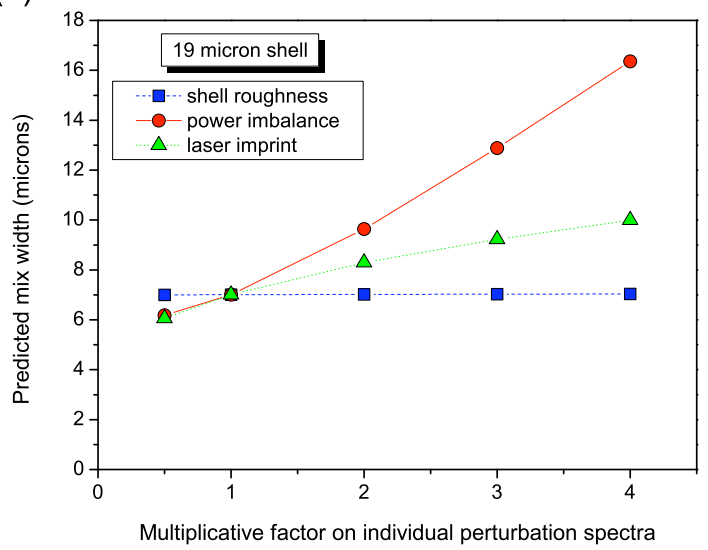

(b)

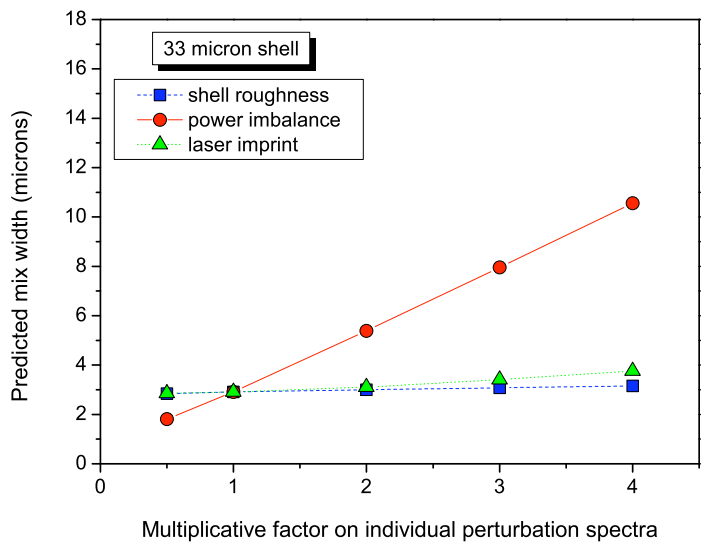

FIG. 4: (Color online) Sensitivity of the Haan mix width to changes in the initial perturbations for the (a) 19 micron and (b) 33 micron shell cases.

added in quadrature, illustrates the relaxation of modes which exceed the saturation curve.

In order to study the sensitivity of the resulting mix width to the individual perturbations, a multiplicative factor was applied to each perturbation one at a time. Figure 4 shows that according to Haan's model, the mix width is not sensitive to changes in the target surface roughness, while it is somewhat sensitive to the laser imprint in the thinner shell case, and it is most sensitive to the power imbalance perturbation.

The laser imprint has a non-negligible effect on the mix layer growth. It has intermediate level amplitudes across a wide band of mode numbers, unlike the other two perturbations, which have significant amplitudes only at small mode numbers. The laser imprint is relatively constant across the shots in a particular day.

The laser beam power imbalance is the largest source of mix sensitivity, due to the fact that the amplitudes at low mode numbers are significantly higher than the 
(a)

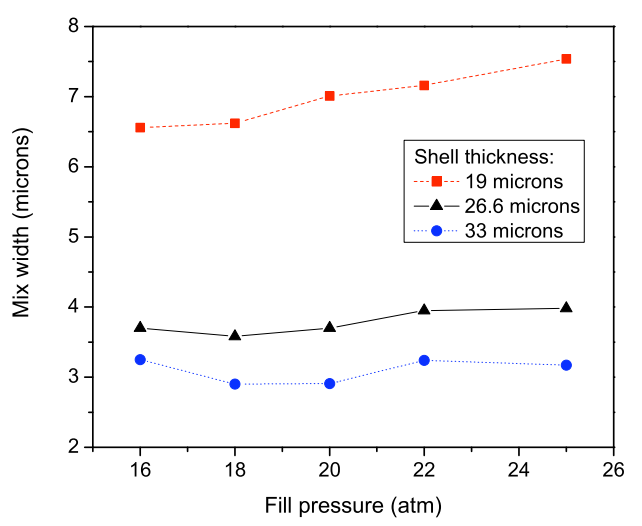

(b)

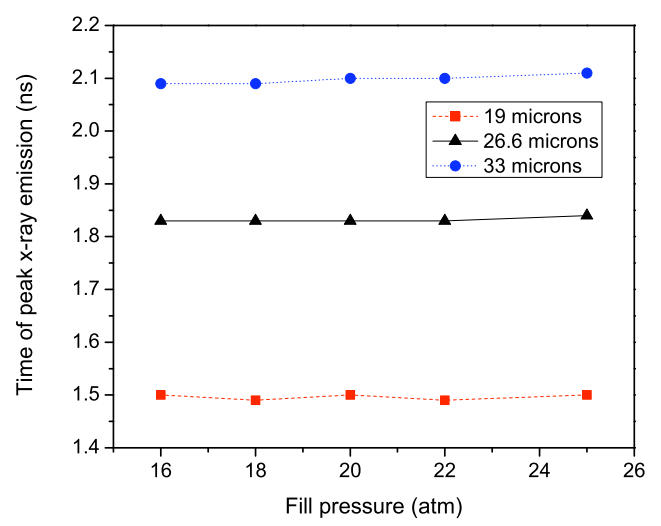

FIG. 5: (Color online) Sensitivity of (a) Haan mix width (at $50 \mathrm{ps}$ after peak x-ray emission) and (b) time of peak x-ray emission to changes in the fill pressure.

other perturbations. This perturbation is probably the largest lever arm in increasing the mix width. The power imbalance is not as easy to control from shot to shot, contributing to uncertainty in the experimentally-derived mix widths.

We concentrate here on unforeseen changes in the experimental parameters which could have a significant effect on the accumulation of clean data that can be used to infer mix widths. The tolerance study focused on varying fill pressures, laser energy, and laser pulse shape.

Figure 5 shows the sensitivity of mix width and time of peak x-ray emission to fill pressure. Assuming that the shell thicknesses are defined to within about a micron, this test demonstrates that according to simulation and post-processing with Haan's model, the mix width is not very sensitive to the fill pressure. The largest change in mix width is exhibited by the 19 micron shell, but even in that case, the mix width only changes by 1 micron.

Figure 6 shows the sensitivity of the mix width and the timing to the total laser energy, which was scaled using (a)

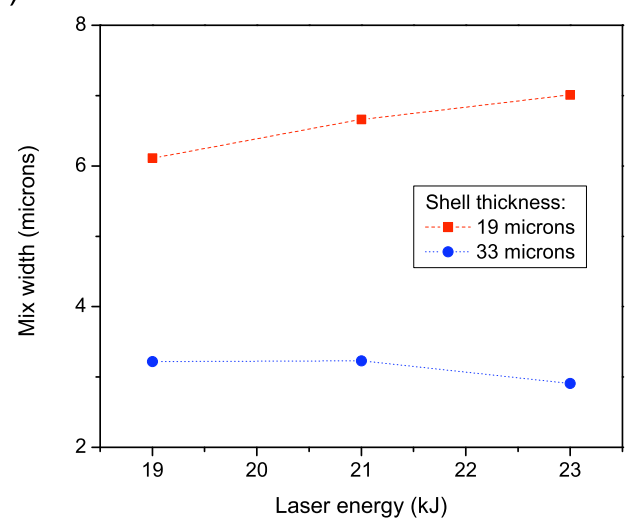

(b)

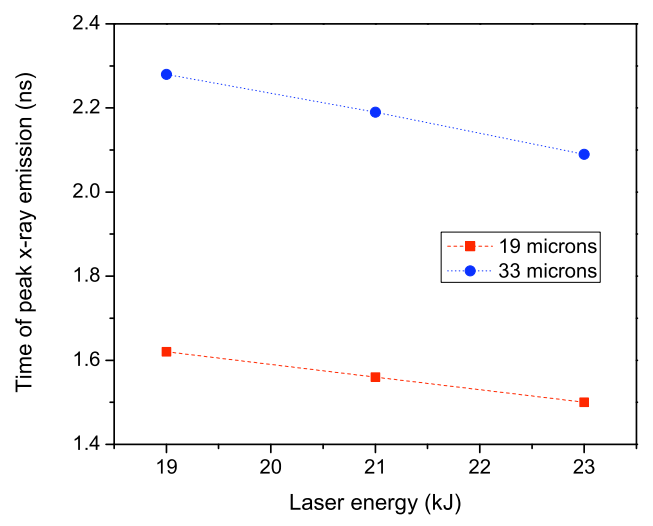

FIG. 6: (Color online) Sensitivity of (a) Haan mix width (at 50 ps after peak x-ray emission) and (b) time of peak x-ray emission to changes in the total laser energy.

the trace in Fig. 1(a). The mix widths are all discernable given the resolution available in the experiment. The time of peak x-ray emission may change substantially due to changes in the laser energy.

A collection of idealized laser pulses was also tested in conjunction with the nominal case. According to this study, the shape of the laser pulse does not significantly impact either the mix width or the x-ray timing.

The conclusion to these tolerance studies was that the major sensitivities in the mix width variation would be a result of either deviation from the planned shell thicknesses or the laser beam power imbalance. The fill pressures, laser energies, and pulse shapes were predicted to not be detrimental in collecting data that could be used for a mix width analysis. However, a substantial change in laser energy should lead to an adjustment of the diagnostic timing in order to accommodate the change in the time of peak-x-ray emission. 
Experiments based on the three shell thicknesses identified in the experimental design phase were fielded at the OMEGA laser. For simplicity, we will refer to the $19 \mu \mathrm{m}$ shell as the thin case, the $26.6 \mu \mathrm{m}$ shell as the nominal case, and the $33 \mu \mathrm{m}$ shell as the thick case.

A total of eight shots were performed: five nominal, two thin, and one thick. Table II lists the experimental characteristics. Generally, the data was good quality, with the thicker shell and a few nominal shells being the best.

For accuracy, new HELIOS-CR hydro simulations were performed to conform to the actual experimental conditions. The experimental laser pulse and energy and actual shell thickness were modified for each simulation. Bang time was matched between simulation and experiment by modifying the flux limiter.

Narrow-band x-ray images of the Ar line emission were recorded with the MMI (Multi-Monochromatic X-ray Imager) [26]. In this case, MMI recorded a series of pinhole sub-images over a spectral energy range encompassing the Ar Ly $\beta$ (1s-3p), Ar He $\beta$ (1 $\left.\mathrm{s}^{2}-1 \mathrm{~s} 3 \mathrm{p}\right)$, and $\operatorname{Ar} \operatorname{Ly} \alpha$ (1s$2 \mathrm{p})$ lines. The MMI data were time-resolved over $50 \mathrm{ps}$. The data recording interval for each case is referenced to $t_{0}$, measured when the laser reaches $2 \%$ of peak power. The experimental timing can therefore be related to simulation timing, since the experimental laser pulse was used in the new simulations.

A graphical user interface tool was built in IDL to accomplish the many steps necessary to process the experimental data. A major benefit of MMI is that it records both line and continuum emission, so continuum images can be subtracted from the line-based images. Figure 7 shows a sequence of narrow-band spatially-resolved images from one of the nominal shots. The images have been corrected for the photon energy dependent instrumental corrections that account for multi-layer mirror reflectivity, Be filter transmission, and multi-channel plate spectral response. These data, along with the associated space-integrated spectrum extracted from performing a lineout across the entire MMI image, form the complete set needed for the experimental data analysis.

TABLE II: Parameters for the eight experimental shots. The bold shot numbers are the data analyzed and presented in Section IV.

\begin{tabular}{|c|c|c|}
\hline Shot & Type & Shell thickness \\
\hline 47470 & nominal & $25.8 \mu \mathrm{m}$ \\
47473 & nominal & $25.6 \mu \mathrm{m}$ \\
47474 & nominal & $25.8 \mu \mathrm{m}$ \\
47476 & nominal & $25.6 \mu \mathrm{m}$ \\
$\mathbf{4 7 4 7 7}$ & nominal & $25.7 \mu \mathrm{m}$ \\
\hline 47481 & thin & $19.3 \mu \mathrm{m}$ \\
$\mathbf{4 7 4 8 4}$ & thin & $19.3 \mu \mathrm{m}$ \\
\hline $\mathbf{4 7 4 8 5}$ & thick & $32.2 \mu \mathrm{m}$ \\
\hline
\end{tabular}

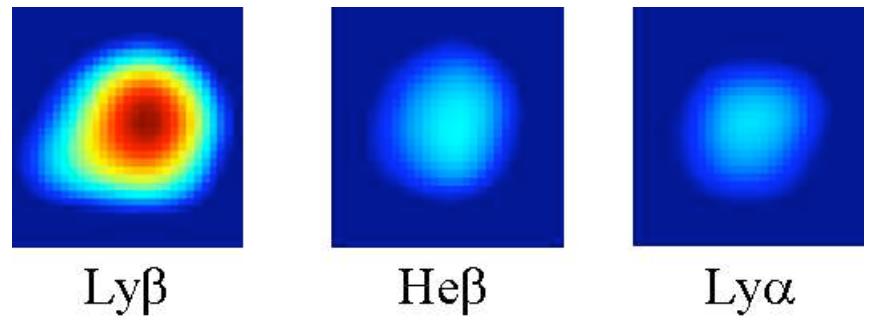

FIG. 7: (Color online) Narrow-band $\operatorname{Ly} \beta, \operatorname{He} \beta$, and Ly $\alpha$ spatially resolved images compiled from MMI data. The analysis of the central columns of data are presented in this paper.

\section{EXPERIMENTAL ANALYSIS}

The spectroscopic data analysis that ultimately allows the extraction of spatial mixing profiles was documented in detail in Refs. $[2,3]$. The same procedures were followed here. We work with the central column of the $\operatorname{Ly} \beta$ and $\mathrm{He} \beta$ narrow-band images, and produce intensity profiles that are symmetrized about a winding axis of symmetry [27]. The intensity profiles are Abel-inverted [28], resulting in emissivity profiles. In this case, both the $\mathrm{He} \beta$ and $\operatorname{Ly} \beta$ can be considered optically thin, so conditions are appropriate to perform an Abel inversion.

The spectroscopic analysis begins with the extraction of a temperature spatial profile using an emissivity ratio technique [2]. The analysis uses the sensitivity of the $\mathrm{Ly} \beta / \mathrm{He} \beta$ emissivity ratio to temperature. A spectroscopic model for $\operatorname{Ar}[29]$ is used to generate the theoretical emissivities, so that the data can be directly tied to theory.

The next step in the spectroscopic analysis is to utilize the intensity technique discussed in Ref. [2]. By using this method, we are able to more correctly account for optical depth effects. The implosion core is idealized as a six-zone nesting set of spheres through which self-emission and transmission terms are added. Figure 8 demonstrates the notion of an idealized core.

Discrete radiation transport equations are set up to calculate the intensity in each spatial zone. For each of the six zones, there exists a system of two equations which link theoretical and experimental intensities and emissivities. The equations include a term which represents, for each zone, the local mixing coefficient $\gamma$, which in this case is the ratio of the atom number densities of plastic shell to fuel material [2]. The simplest set of equations is for the outer zone 0 , which has only a selfemission term in the intensity equation:

$$
\begin{gathered}
I_{L y \beta}^{e x p}(0)=k^{\prime} \frac{\epsilon_{L y \beta}^{\text {theo }}(0)}{\kappa_{L y \beta}^{\text {theo }}(0)}\left[1-e^{-\frac{1}{(1+\zeta \gamma(0))} \kappa_{L y \beta}^{\text {theo }}(0) L(0,0)}\right] \\
\epsilon_{H e \beta}^{\exp (0)}=\frac{k}{(1+\zeta \gamma(0))} \epsilon_{H e \beta}^{\text {theo }}(0) .
\end{gathered}
$$

The constant $\zeta$ in front of the mixing coefficient $\gamma$ is a factor related to the fill pressure of the gas [2]. The $L$ 


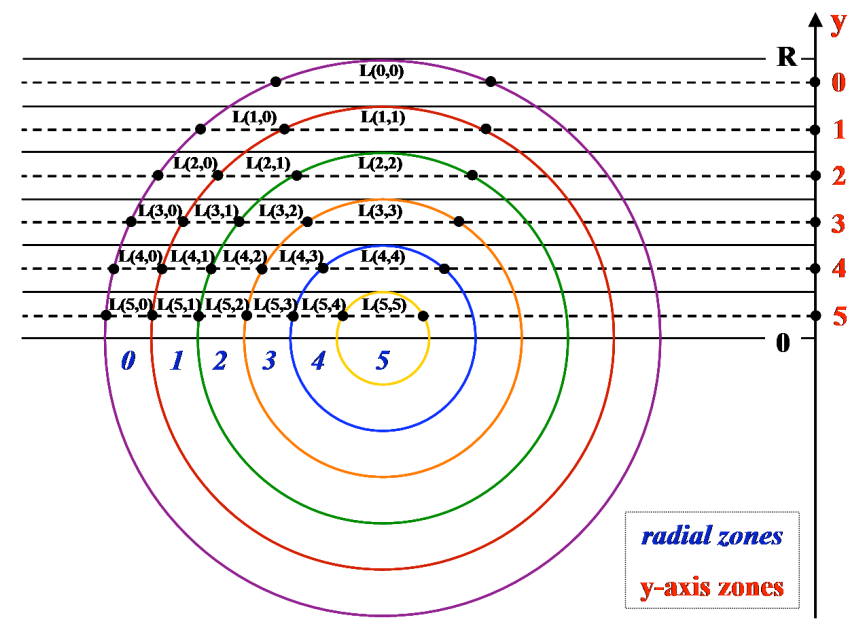

FIG. 8: (Color online) Idealization of the implosion core for the solution of discrete radiation transport equations.

represents the length of a chord, as shown in Fig. 8. The $\kappa^{\text {theo }}$ term in the intensity equation is the opacity from the spectral model, which is a function of the electron density $N_{e}$ and the previously calculated electron temperature $T_{e}$. The $\epsilon^{\text {theo }}$ terms are the emissivities from the model, which are also functions of $T_{e}$ and $N_{e}$. Finally, the $k$ and $k /$ represent the proportionality constants between the theoretical values for the emissivity and intensity of a specific zone and the experimental values recorded in the data. A discussion of the calculation of $k$ and $k /$ is included in Ref. [2]. The set of two equations for zone $r$ (zone $r=0$ is shown in Eqns. 8 and 9) have only two unknowns, the $N_{e}(r)$ and $\gamma(r)$. A caveat is that we must solve the equations from the outside zone inward, since each subsequent inner zone relies on information from the outer zones.

We focus here on the mixing spatial profiles generated from these equations as applied to the data discussed in Section III. Figure 9 displays the mix profiles for nominal, thin, and thick shell cases. The uncertainty bars in the data points represent a weak density sensitivity in the emissivity analysis used to calculate the temperatures, which then carries through to the density and mix calculations. Two types of fits are shown for different purposes. Exponentials of the form $\gamma=A e^{\frac{x}{x_{0}}}+\gamma_{0}$, where $x_{0}$ is the characteristic width of the exponential and $\gamma_{0}$ is a term representing the intersection of the fit with the $\gamma$ axis, fall within the error bars of all data points, but indicate that there is a slight amount of mixing in the center of the core. Exponentials of the form $\gamma=A e^{\frac{x}{x_{0}}}$, where there is no offset on the $\gamma$ axis at the center, were employed specifically to match the results of Haan's model, which suggest that there is no mixing at the center in these cases.

The thick and nominal data were of sufficient quality to extract information out of the outer five zones in the problem. The thin data, however, was not as good qual-

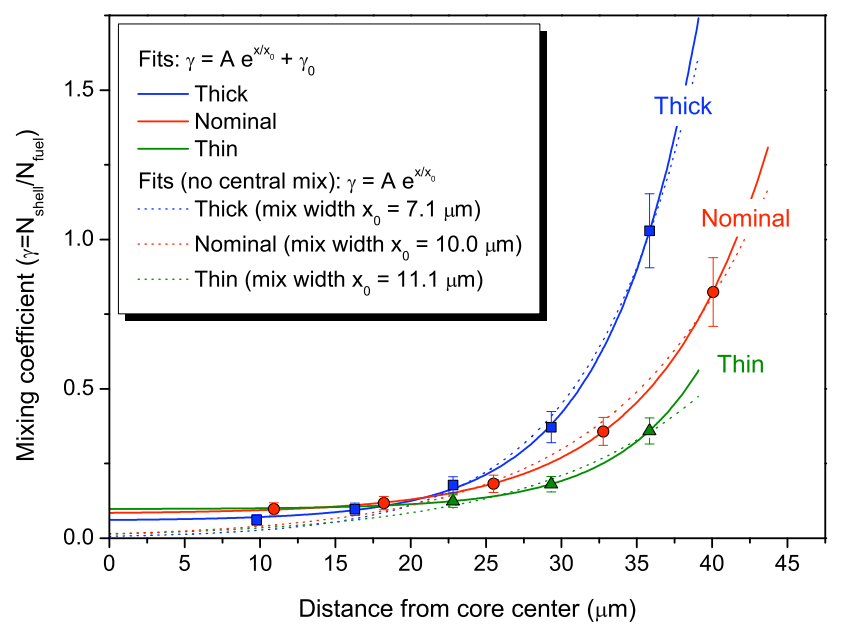

FIG. 9: (Color online) Spatial mix profiles for the nominal, thin, and thick shell cases. Solid fits represent no assumptions made about mixing in the center of the core, while the dotted fits are representative of assuming no mix in the center.

ity, possibly due to a slight amount of burn-through in the shell. In this case, information could be extracted from only three of the six zones, leaving open the interpretation of the inner half of the thin mixing profile.

\section{COMPARISON OF THEORETICAL DESIGN AND EXPERIMENTAL ANALYSIS}

The mixing information gathered from the hydro simulations in conjunction with the mix models is in the form of a single number representing the mix width. However, the experimental data analysis gives more detail, in the form of a spatial mixing profile. It is necessary to reconcile these two descriptions of mixing in order to correctly compare theory and experiment. We begin this discussion by pointing out several subtleties in the way we measure mix.

The theoretical framework relies on Haan's saturation model, which measures the width of the bubble and spike regions independently. The sum of the bubble and spike widths gives the total width of the mix region. The bubbles and spikes grow on either side of the interface between the fuel and shell materials, as indicated in Fig. 10.

In a strictly hydrodynamic sense, the core size at a specific time is generally considered to be the position of the interface at that time. However, this description only relates to a clean simulation that does not experience mix. By calculating the width of the mix region surrounding the fluid interface, we are in effect viewing a prediction of what a real (mixed) experiment would look like. In an experiment, the core size viewed in an x-ray image would include not only the spike but also the bubble region outside the interface. Recall that the bubble is comprised of fuel material, which in this case contains $\mathrm{D}_{2}$ gas plus the 


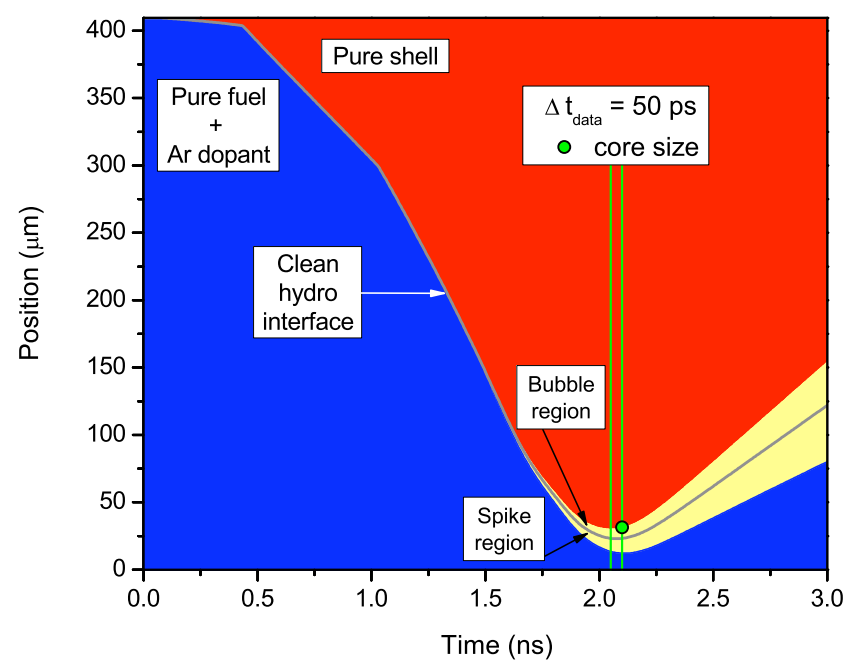

FIG. 10: (Color online) Mix width definition from a hydro point of view, with the bubble region above and the spike region below the interface. The blowup shows the concept of the bubbles and spikes spanning the interface. The green vertical lines represent the 50 ps time interval over which the framing camera recorded data in this example. The core size prediction based on this hydro data should therefore be the maximum position of the top of the bubble region throughout this 50 ps time interval, since this is consistent with the core size visualized in the data.

Ar dopant. The Ar line emission is what is being imaged experimentally, and therefore in order to effectively compare the measurements from the experiment to the predictions of the hydro, we must define the total core size as the maximum of the 50 ps region of integration for the experimental data. Figure 10 shows an example of this time interval and the corresponding core size that is physically consistent with the core size extracted from the data. Figure 11 shows an example of the intensity profile of Ar Ly $\beta$ line emission, and again we emphasize that the core size at the time of data recording includes not only the pure fuel region but also the bubbles and spikes, i.e. the entire mixed region.

According to the preceding arguments, we are careful to paint a consistent picture when relating the core size predicted by the simulation (Fig. 10) to the core size seen in the data (Fig. 11). However, despite these efforts, the core sizes do not match well. The 1d simulations tend to over-compress the targets, resulting in smaller core sizes than is seen in the data. In order to dispel this issue, we focus on the percentage of the core that is mixed rather than on the actual mix width and core size values. This enables us to maintain consistency between the theoretical and experimental points of view.

As demonstrated in Fig. 9, the experimental data analysis does not result in mix widths, which can be directly compared to the theoretical predictions, but in mix profiles. The fits of the form $\gamma=A e^{\frac{x}{x_{0}}}+\gamma_{0}$ shown in Fig. 9 introduce a $\gamma$ offset which represents the idea that

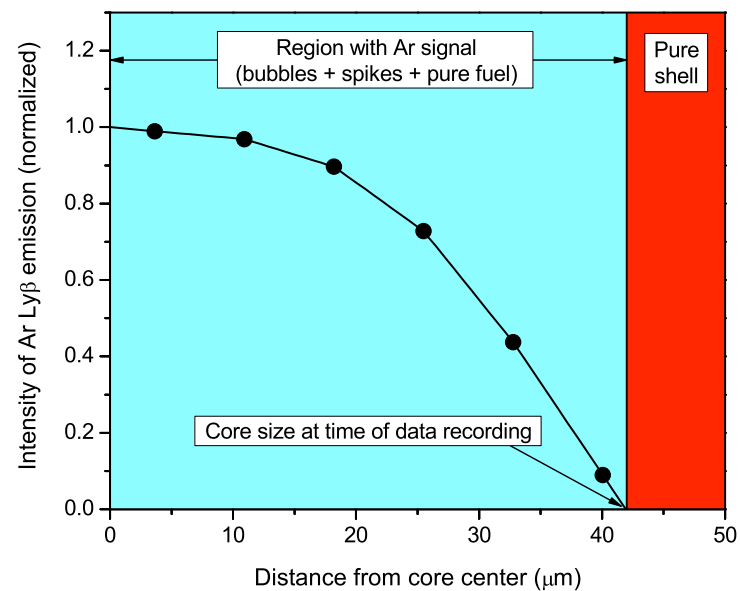

FIG. 11: (Color online) Example of an Ar Ly $\beta$ intensity spatial profile extracted from the data, detailing the core size seen by the imager at the time the data were recorded. Note that the core size in this context represents the total width of any pure fuel plus the bubble and spike widths.

the mixing coefficient might not necessarily be zero at the core center. However, our implementation of Haan's model does calculate $\gamma=0$ at the center, since the mix width is always smaller than the core size. As mentioned in the previous section, we therefore compress the experimentally derived mix profiles down to mix width estimations by employing the exponential form $\gamma=A e^{\frac{x}{x_{0}}}$, which removes the $\gamma$ offset and effectively provides a mix width through the characteristic width of the exponential, $x_{0}$. These modified mixing profile fits are within the error bars of the outer four data points for the nominal and thick profiles, and within all three data points for the thin profile.

Table III presents a comparison between the mix calculated by the simulations (which were modified to account for experimental conditions) and the mixing information extracted from the experimental data. The core sizes and mix widths do not necessarily match those shown in the predictions of $\mathrm{Tbl}$. I, since those simulations were based on generic laser pulses, shell thicknesses, and timing estimates of 50 ps after peak x-ray emission. The data were actually captured approximately 120 ps after peak x-ray emission (relative to the simulation timing), resulting in different estimations for the core sizes and mix widths.

Note that the Haan multiplier used to generate the mix widths shown in Tbl. III was the nominal multiplier of 1.0. Previous work in an indirect drive platform required a Haan multiplier of 2.7 to match the data [4]. We conclude that, as others have noted [30], the nominal Haan saturation model coupled with the calculation of growth factors tends to be more applicable to direct drive than to indirect drive.

Though the actual core sizes and mix widths do not 
TABLE III: Comparison between mix information calculated by post-processing simulations with Haan's saturation model and mix data extracted from the experiments. For the simulations, the core size is the interface position plus the bubble width at the maximum position over the interval of data recording, while the Haan mix width is the sum of the bubble and spike widths. For the experiments, the core size is the edge of the core as defined by the spatial limit of Ar emission, and the mix width is extracted from a fit to $\gamma=A e^{\frac{x}{x_{0}}}$.

\begin{tabular}{|l|c|c|c|c|c|c|}
\hline \multirow{2}{*}{ Shot } & \multicolumn{3}{|c|}{ Simulation } & \multicolumn{3}{c|}{ Experiment } \\
\cline { 2 - 7 } & Core size & Haan mix width & of core mixed & Core size & Experimental mix width $\%$ of core mixed \\
\hline Thin $(47484)$ & $34.4 \mu \mathrm{m}$ & $18.0 \mu \mathrm{m}$ & $52 \%$ & $39.1 \mu \mathrm{m}$ & $11.1 \mu \mathrm{m}$ & $28 \%$ \\
\hline Nominal $(47477)$ & $26.3 \mu \mathrm{m}$ & $6.8 \mu \mathrm{m}$ & $26 \%$ & $43.7 \mu \mathrm{m}$ & $10.0 \mu \mathrm{m}$ & $23 \%$ \\
\hline Thick $(47485)$ & $26.7 \mu \mathrm{m}$ & $3.5 \mu \mathrm{m}$ & $13 \%$ & $39.1 \mu \mathrm{m}$ & $7.1 \mu \mathrm{m}$ & $18 \%$ \\
\hline
\end{tabular}

compare well between simulation and experiment, the trends in the percentage of the core that is mixed are clear. According to both the simulations and the data, a larger portion of the thin shell core is mixed, while the thicker shell core experiences the least amount of mix. This behavior can also be seen qualitatively in the mix profiles of Fig. 9, where the thin profile tends to drop considerably more slowly than do the nominal and thick profiles. These trends are consistent with the predictions made in the design phase of this experimental campaign.

\section{SUMMARY}

The main goal of this work was to design and subsequently perform a series of direct drive ICF implosions which exhibit measurably different levels of mix. In the experimental design phase, $1 \mathrm{~d}$ hydrodynamic simulations were post-processed with Haan's saturation model. A parameter study involving a number of experimental characteristics was performed, and it was found that of the parameters investigated, the shell thickness of an ICF capsule is most sensitive to mix according to Haan's model.

Consequently, three types of experiments were performed at the OMEGA laser facility. The shots were nominally identical except for varying initial shell thicknesses of $\sim 19,27$, and 33 microns. The MMI instrument provided the narrow-band spatial images and spectra necessary to extract mixing information through a spectroscopic analysis which was previously tested on in- direct drive experiments. The narrow-band image intensity analysis resulted in spatial mixing profiles for the thin, nominal, and thick shells. The portion of the core that is mixed was extracted from these mixing profiles, and compared to the comparable values derived from Haan's saturation model as applied to the hydro simulations. We found good agreement between data and theory in the trends, which demonstrated that thinner shells develop significantly more mix than thicker shells.

Spectroscopic analysis of the experimental data demonstrates that we have developed a reasonable predictive capability for mix in ICF implosions. The mix models were used as a guide to design the experiments, which were in turn used to check the applicability of the mix models. In essence, we have self-consistently tested not only the spectroscopic methods used to extract mixing information directly from experimental data, but also the applicability of the Haan mix model to direct drive ICF implosions.

\section{Acknowledgments}

This work was performed under the auspices of the U.S. DOE/NNSA Campaign 10 at Los Alamos National Laboratory. The authors wish to acknowledge the support of DOE-NLUF grant DE-FG52-2005NA26012, which provided the experimental campaign. Work was also supported by Lawrence Livermore National Laboratory under Contract DE-AC52-07NA27344.
[1] J. Lindl, Phys. Plasmas 2, 3933 (1995).

[2] L. Welser-Sherrill, R. Mancini, J. Koch, N. Izumi, R. Tommasini, S. Haan, I. Golovkin, J. MacFarlane, J. Delettrez, F. Marshall, et al., Phys. Rev. E 76, 056403 (2007).

[3] L. Welser-Sherrill, R. Mancini, J. Koch, N. Izumi, R. Tommasini, S. Haan, D. Haynes, I. Golovkin, J. Delettrez, S. Regan, et al., High Ener. Dens. Phys. 3, 287 (2007).

[4] L. Welser-Sherrill, R. Mancini, D. Haynes, S. Haan, J. Koch, N. Izumi, R. Tommasini, I. Golovkin, J. Mac-
Farlane, J. Delettrez, et al., Phys. Plasmas 14, 072705 (2007).

[5] T. Boehly, D. Brown, R. Craxton, R. Keck, J. Knauer, J. Kelly, T. Kessler, S. Kumpan, S. Loucks, S. Letzring, et al., Opt. Commun. 133, 495 (1997).

[6] D. Youngs, Physica D 12, 32 (1984).

[7] S. Haan, Phys. Rev. A 39, 5812 (1989).

[8] L. Welser-Sherrill, D. Haynes, R. Mancini, J. Cooley, S. Haan, and I. Golovkin, AIP Conf. Proc. CP926, 238 (2007).

[9] Since we are using Ar spectroscopy as a tool to diagnose 
the plasma conditions, the experimental design is constrained to result in temperatures and densities which are consistent with the sensitivity range of Ar x-ray line emission.

[10] The experimental data are recorded over a 50 ps time interval coinciding with peak x-ray emission from the $\mathrm{Ar}$ dopant. Therefore, in the experimental design phase, mix widths and other quantities were extracted at $50 \mathrm{ps}$ after peak $\mathrm{x}$-ray emission in order to be consistent with the planned data recording time.

[11] R. Mancini, C. Hooper, N. Delamater, A. Hauer, C. Keane, B. Hammel, and J. Nash, Rev. Sci. Instrum. 63, 5119 (1992).

[12] H. Griem, Phys. Fluids B 4, 2346 (1992).

[13] B. Hammel, C. Keane, M. Cable, D. Kania, J. Kilkenny, R. Lee, and R. Pasha, Phys. Rev. Lett. 70, 1263 (1993).

[14] D. Haynes, C. Hooper, R. Mancini, D. Bradley, J. Delettrez, R. Epstein, and P. Jaanimagi, Rev. Sci. Instrum. 66, 755 (1995).

[15] D. Haynes, D. Garber, C. Hooper, R. Mancini, Y. Lee, D. Bradley, J. Delettrez, R. Epstein, and P. Jaanimagi, Phys. Rev. E 53, 1042 (1996).

[16] N. Woolsey, A. Asfaw, B. Hammel, C. Keane, C. Back, A. Calisti, C. Mosse, R. Stamm, B. Talin, J. Wark, et al., Phys. Rev. E 53, 6396 (1996).

[17] N. Woolsey, B. Hammel, C. Keane, C. Back, J. Moreno, J. Nash, A. Calisti, C. Mosse, L. Godbert, R. Stamm, et al., J. Quant. Spectrosc. Radiat. Transfer 58, 975 (1997).
[18] N. Woolsey, B. Hammel, C. Keane, A. Asfaw, C. Back, J. Moreno, J. Nash, A. Calisti, C. Mosse, R. Stamm, et al., Phys. Rev. E 57, 4650 (1998).

[19] S. Regan, J. Delettrez, R. Epstein, P. Jaanimagi, B. Yaakobi, V. Smalyuk, F. Marshall, D. Meyerhofer, W. Seka, D. Haynes, et al., Phys. Plasmas 9, 1357 (2002).

[20] H. Griem, Principles of Plasma Spectroscopy (Cambridge University Press, 1996).

[21] B. Yaakobi, F. Marshall, and R. Epstein, Phys. Rev. E 54, 5848 (1996).

[22] J. MacFarlane, I. Golovkin, and P. Woodruff, J. Quant. Spectrosc. Radiat. Transfer 99, 381 (2005).

[23] J. MacFarlane, I. Golovkin, R. Mancini, L. Welser, J. Bailey, J. Koch, T. Mehlhorn, G. Rochau, P. Wang, and P. Woodruff, Phys. Rev. E 72, 066403 (2005).

[24] L. Welser-Sherrill, J. Cooley, and D. Wilson, Comp. Phys. Comm submitted for publication (2008).

[25] The value of $\nu=2$ is consistent with $\alpha_{b}=0.04$, as is currently used in ICF applications.

[26] J. Koch, T. Barbee, N. Izumi, R. Tommasini, R. Mancini, L. Welser, and F. Marshall, Rev. Sci. Instrum. 76, 073708 (2005).

[27] J. Koch, T. Barbee, S. Dalhed, S. Haan, N. Izumi, R. Lee, L. Welser, R. Mancini, F. Marshall, D. Meyerhofer, et al., AIP Conf. Proc. 730, 53 (2005).

[28] K. Bockasten, J. Opt. Soc. Am. 51, 943 (1961).

[29] R. Mancini, Private communication.

[30] S. Haan, Private communication. 